\title{
КЛАССИФИКАЦИЯ ПАРАМЕТРОВ И ПОКАЗАТЕЛИ КАЧЕСТВА ВЫБОРА ЭЛЕМЕНТОВ РЕНТГЕНООПТИЧЕСКОЙ СИСТЕМЫ
}

\begin{abstract}
С.С. Баус, И.В. Плотникова, Н.В. Чичерина
Научно-образовательный процесс сегодня должен представлять систему, в которой формируется представление об изменяющемся мире, где закрепляются и передаются традиции ответственности перед будущими поколениями за предложенные инженерные решения. В настоящее время при проектировании новых модифрикаций рентгеновских томографоов встает задача подбора значений некоторых параметров, таких как: напряжения на рентгеновской трубке и тип исследуемого материала. Для сокращения времени и трудоемкости проектирования необходимо определить основные параметры и характеристики элементов. В работе большее внимание уделяется ключевым элементам рентгенооптической системы - рентгеновской трубке, детектору рентгеновского излучения, а также геометрических и пространственных размеров самой рентгенооптической системы, так как от выбора правильного расстояния зависит конечное качество теневого изображения, в частности, его резкость, четкость, оптимальный масштаб, возможность воссоздать трехмерное изображение. Определены основные параметры качества изображения, полученных с помощью цифрровых детекторов. Получены ключевые характеристики, основные зависимости параметров, показатели качества, а также рекомендации по выбору элементов рентгеновских систем. Показана зависимость размера вокселя детектора от изменения значений расстояний от рентгеновской трубки до оси вращения объекта.

Ключевые слова: классификация, параметры, характеристика, элементы, излучение, рентеенооптическая система.
\end{abstract}

\section{ВВЕДЕНИЕ}

В современном мире для промышленного неразрушающего контроля наиболее распространёнными системами являются рентгеновские томографы, которые разделяются по нескольким типам: размерам исследуемых величин, техническим характеристикам, области применения и т. д. Популярным направлением развития данных систем можно считать микротомографы [1]. Данные системы позволяют исследовать внутреннюю структуру предметов с микронными размерами. Данные системы характеризуются напряжением на рентгеновской трубке от 10 до 160 кВ, маленьким размером фокусного пятна - от 1 до 10 мкм. Поскольку бетатроны относятся уже к другому классу рентгеновских систем за счет иного принципа реализации и технических характеристик в данной работе они не рассматриваются.

На данный момент на рынке представлено четыре компании, которые предлагают микротомографы, отвечающие современным передовым требованиям в области энергосбережения, фуннкциональных характеристик, точности измерения и пучка рентгеновского излучения. Это Matrix (Великобритания), Sky-
Scan (Бельгия), Feinfocus (Германия), Fraunhofer (Германия).

При создании новых систем рентгеновского томографа этап проектирования является одним из основополагающих. На этой стадии рассчитываются основные параметры технической системы в соответствии с техническим заданием, разрабатываются принципиально новые технические решения, производится компоновка системы.

Деятельность по разработке и проектированию рентгеновских систем имеет опытный характер, выбор комплектации и параметров системы производится с помощью теоретических формул [2], а также интуитивного подбора комплектующих, которые соответствуют данным параметрам [3]. Перед научными сотрудниками и инженерами всегда встает острый вопрос о выборе элементов рентгенооптической системы [4]. При проектировании новых систем, прежде всего, руководствуются следующими составляющими: технические параметры элементов системы, видом и режимами работы детектора, размер и вид исследуемого материала $[5,6]$, требования к точности [7], себестоимостью, допустимые условия работы [8], энергопотребления. 


\section{ОСНОВНАЯ ЧАСТЬ}

Рассмотрим, что представляет рентгеновская система. Рентгенооптическая система, предназначенная для неразрушающего контроля, состоит из четырех элементов: рентгеновской трубки, детектора рентгеновского излучения, системы позиционирования рабочего стола, исследуемого материала [9].

В данной статье большее внимание уделяется именно двум ключевым элементам рентгенооптической системы - рентгеновской трубке, детектору рентгеновского излучения, а также геометрических и пространственных размеров самой рентгенооптической системы. Ведь от выбора правильного расстояния зависит конечное качество теневого изображения, в частности, его резкость, четкость, оптимальный масштаб, возможность воссоздать трехмерное изображение.

Как было сказано выше, основным элементом рентгенооптической системы является рентгеновская трубка. Рассмотрим ее поподробнее. Рентгеновская трубка - электровакуумный прибор с источником излучения электронов (катод) и мишенью, в которой они тормозятся (анод) [8]. Основными техническими характеристиками источников рентгеновского излучения являются:

- тип рентгеновской трубки;

- диапазон напряжений, кВ;

- шаг выбора напряжений, кB;

- диапазон токов на аноде, мА;

- максимальная мощность на аноде, Вт;

- мощность рентгеновской трубки, Вт;

- размер фокального пятна, мм;

- угол выхода излучения, градус.

Ключевые характеристики рентгеновских трубок, определяющие их возможности:

- фокусирующие элементы - это электромагнитные или другие элементы внутри трубки, которые «втискивают» ускорившиеся электроны в настолько маленькую точку на мишени, насколько это возможно. Эта точка на мишени называется фокальным пятном. Чем меньше фокальное пятно, тем лучше разрешение конечного изображения;

- размеру фокального пятна трубки делятся на 2 типа: микрофоокусные и нанофокусные. Размер фрокусного пятна в микрофрокусных трубках составляет Змкм;

- вид и тип мишени. Основными видами на данный момент являются прострельная и отражательная. Прострельная мишень характеризуется тем, что рентгеновские лучи должны пройти сквозь саму эту мишень. Если же использовать отражательную мишень, то испускаемое излучение отражается от поверхности мишени. Тип мишени оказывает непосредственное влияние на размер теневого изображения [10]. Также стоит отметить, что минимальное расстояние между фокальным пятном и исследуемым материалом для различных типов мишеней различен. Для прострельной мишени - это величина равняется до 0,6 мм. Для отражательной же мишени, которая зачастую установлена в закрытую трубку - 12-16 мм;

- материал мишени и толщина этого материала. Это особенно важно для прострельной мишени, так как для коммерческого использования (то есть для повышения срока службы) нужна хорошая плотность потока рентгеновского излучения и в то же время несильное его поглощение при прохождении через мишень. Чаще всего для мишени используется вольфрам.

Длина волны рентгеновского излучения определяет его энергию, т.е. способность проникать внутрь объекта. Рентгеновское излучение с более короткой длиной волны, образуемое при более высоком значении кВт, обладает большей проникающей способностью по сравнению с рентгеновским излучением с большей длиной волны (менее энергетичное излучение). Важным нюансом стоит учитывать, что в рентгеновских трубках с напряжением до 60 кВ только $0,1 \%$ энергии преобразуется в энергию рентгеновского излучения. При напряжении 100 кВ КПД трубки увеличивается до $1 \%$.

Рассмотрим поведение рентгеновского излучения. Объект исследования является самым важным источником данных для определения таких параметров, как энергия рентгеновского излучения (напряжение на трубке), тип источника рентгеновского излучения, толщина защитных свинцовых экранов (от рассеянного) и схема просвечивания. Все эти параметры выбираются в зависимости от геометрических размеров контролируемого изделия, чтобы чувствительность контроля не превышала половины размера по глубине минимального из недопустимых дефектов. На рисунке 1 изображена зависимость толщины просвечиваемого материала от напряжения на рентгеновской трубке. Исследуемым материалом в данном случае является железо, рентгеновская трубка Hammatsu.

Данные значения получены эмпирическим путем. Это крайне важно. Ведь деятельность по определению оптимальных параметров системы имеет опытный характер, выбор комплектации и режимов системы производится с помощью теоретических 
формул, а также интуитивности настройки. Данные расчеты характеризуют лишь ситуативное (статическое) состояние рентгенооптической системы и не позволяют в полной мере оценить поведение системы (изменение выходного параметра) при изменении значений факторов.

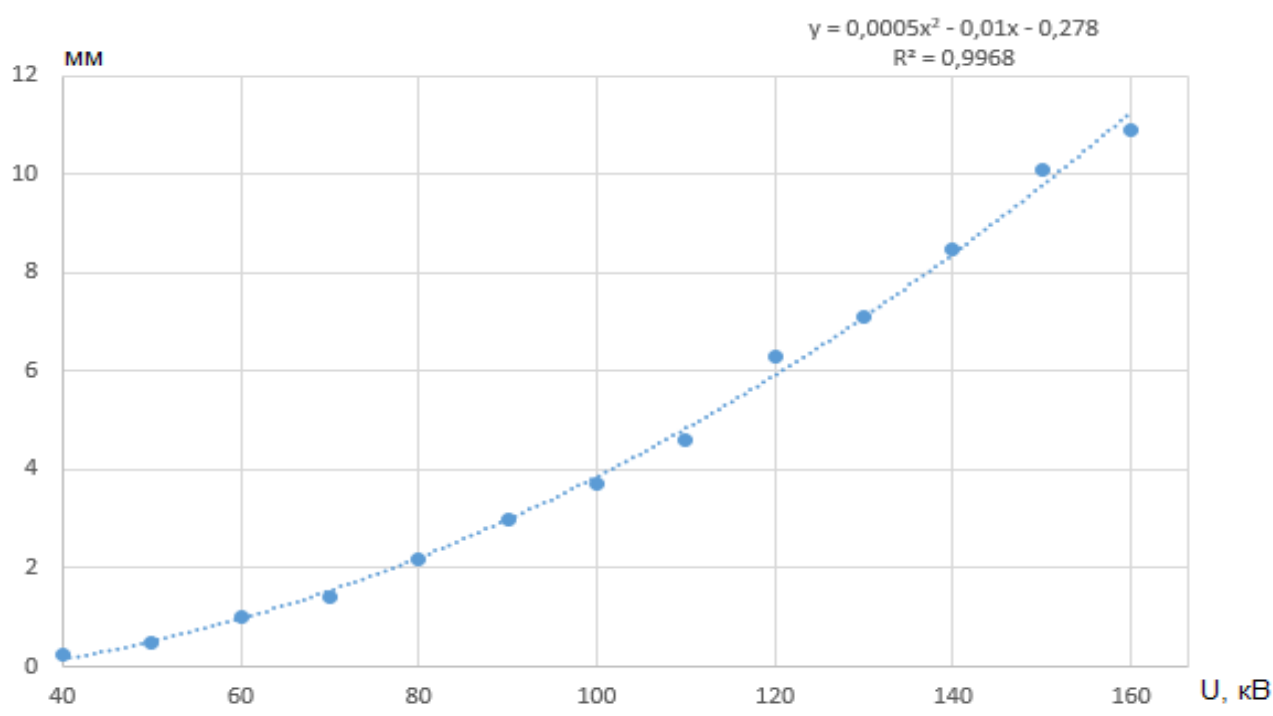

Рисунок 1 - Зависимость толщины просвечиваемого материала от напряжения

Это проблема решается с помощью математической зависимости.

Как видно из рисунка 1 данная зависимость имеет форму кривой и описывается уравнением $\quad y=0.0005 x^{2}+0.01 x+0.278$. Доля данного фрактора 99,68\% - это говорит нам о том, что уравнение имеет очень высокие показатели точности.

Не менее важную роль в рентгенооптической системе отводится детектору рентгеновского излучения, а также расстоянию между рентгеновской трубкой, исследуемым материалом и детектором. В подавляющем большинстве компьютерных рентгеновских томографов используются цифровые детекторы. Рентгеновский цифровой детектор - это устройство, которое предназначено для получения цифровых рентгеновских изображений.

Цифровые детекторы для рентгена имеют следующие основные технические характеристики [11]:

- $\quad$ размер активной области детектора

- фоктор оптической восприимчивости поверхности детектора

изображений

- предельное пространственное разрешение

- $\quad$ размер матрица изображения

- градационная разрешающая способность.
Основными параметрами качества изображения, полученных с помощью цифровых детекторов. К ним можно отнести:

- нерезкость полученных снимков;

- соотношение контраста к шуму;

- соотношение сигнала к шуму;

- влияние на величину эффективного пикселя матрицы;

- контрастная чувствительность [12];

- пространственное разрешение.

Нежелание заказчиков платить астрономические суммы за детекторы большого размера. Увеличение физических размеров детекторов ведёт к увеличению размера полупроводниковой светочувствительной матрицы, а это всегда влияет на стоимость. Производители по-разному пытаются разрешить это противоречие. К примеру, в иногда детектор перемещается вдоль образца [13]. Полученные части изображения затем «сшиваются» в единую 3D-модель с помощью специализированного программного обеспечения. Несмотря на кажущуюся простору, это связано с некоторыми техническими трудностями. Для успешной сшивки изображения требуется прецизионная система перемещения детектора. И снова приходится констатировать, что оснащение томографа подобной системой перемещения детектора отнюдь не приводит к уменьшению её стоимости.

Размер исследуемого материала и качество снимка, полученного с помощью детек- 


\section{КЛАССИФИКАЦИЯ ПАРАМЕТРОВ И ПОКАЗАТЕЛИ КАЧЕСТВА ВЫБОРА ЭЛЕМЕНТОВ РЕНТГЕНООПТИЧЕСКОЙ СИСТЕМЫ}

тора, напрямую зависит от расстояний рентгенооптической системы, в частности от пар рентгеновская трубка-детектор, трубкаобъект, объект-детектор. Для расчета этих параметров произведем следующие расчеты.

Для анализа были выбраны девять цифровых рентгеновских томографов, которые представлены на рынке: Hammatsu, GEDXR250, GE DXR250RT, Perkin Elmer XRD0840, Varian PaxScan 1313, Toshiba
E5877J, Toshiba E5876J, SkyScan 210, RadEye200.

Из рисунка 2 можно определить, что зависимость ширины объекта от расстояний от трубки до оси вращения объекта видоизменяется по линейному закону. В итоге, чем больше значение длины объекта при фриксированном расстоянии, тем шире диапазон исследуемых величин, что делает детекторы с данными параметрами лучше конкурентов.

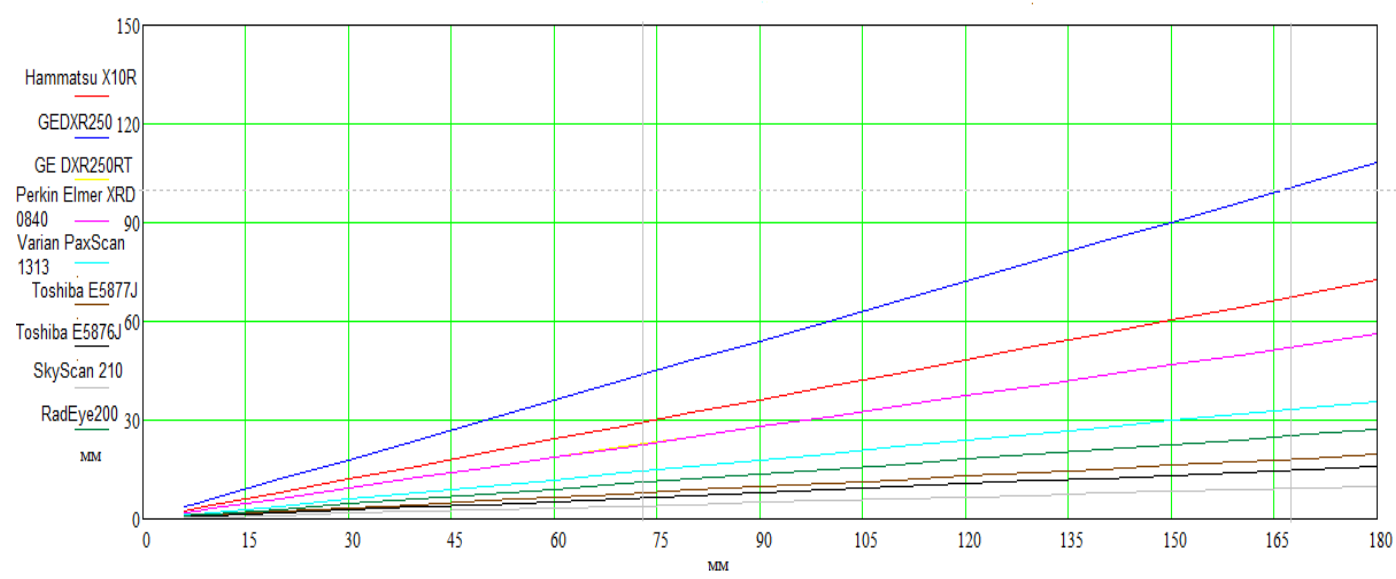

Рисунок 2 - Зависимость размера объекта от расстояния до оси вращения

Математически проекционное увеличение $M$ можно вычислить по следующим отношениям:

$$
M=\frac{D_{F}}{D_{o}}=\frac{d_{f}}{d_{o}},
$$

где $d_{0}$ - расстояние от фокусного пятна рентгеновской трубки до объекта, $d_{f}$ - расстояние от фокусного пятна рентгеновской трубки до детектора, $D_{o}$ - размер объекта исследования; $D_{f}$ - проекционное увеличение объекта исследования.

Из рисунка 3 наглядно видно, что геометрическое увеличение уменьшается по гиперболическому закону с возрастанием значений расстояния от трубки до оси объекта.

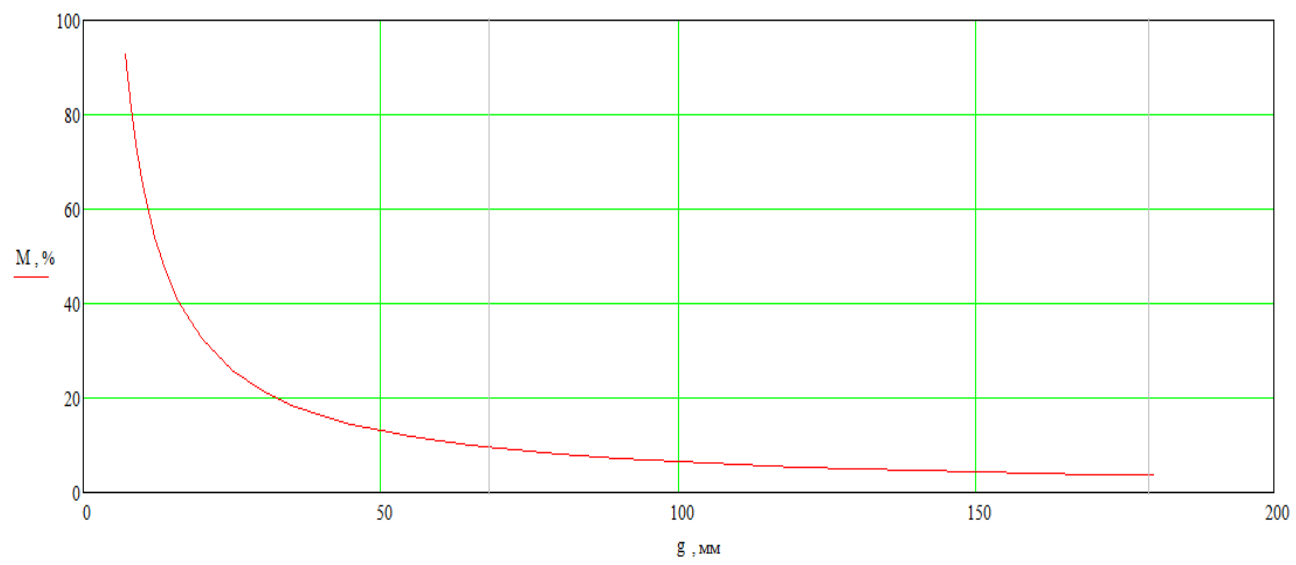

Рисунок 3 - График геометрического увеличения от расстояний от трубки до объекта

Размер вокселя рассчитывается как отношение размера пикселя к геометрическому увеличению $\left(M=\frac{H}{g}\right): v=\frac{p}{M}=\frac{p g}{H}$
Из рисунка 4 можно определить, размер вокселя детектора изменяется по линейному закону в зависимости от изменения значений расстояний от рентгеновской трубки до оси вращения объекта. Лучшие показатели из 
приведенных в данном расчете детекторов показала фирма SkyScan и Hammatsu. Размер вокселя важная характеристика детектора для получения теневых tiff-изображений и последующей трехмерной реконструкции.

Она определяет качество снимка, его детализацию. Поэтому крайне важно правильно спроектировать и выбрать размеры рентгенооптической системы. Фокусное расстояние следует выбирать таким образом, чтобы геометрическая нерезкость была со- измерима со значением внутренней нерезкости. Увеличение фокусного расстояния позволяет уменьшить нерезкость, но при этом снижается интенсивность излучения и увеличивается время экспозиции. В рентгеновских трубках малое фокальное пятно позволяет получить лучше разрешение. Чем пятно больше, тем больше размыты контуры и больше ограничено разрешение получаемого изображения.

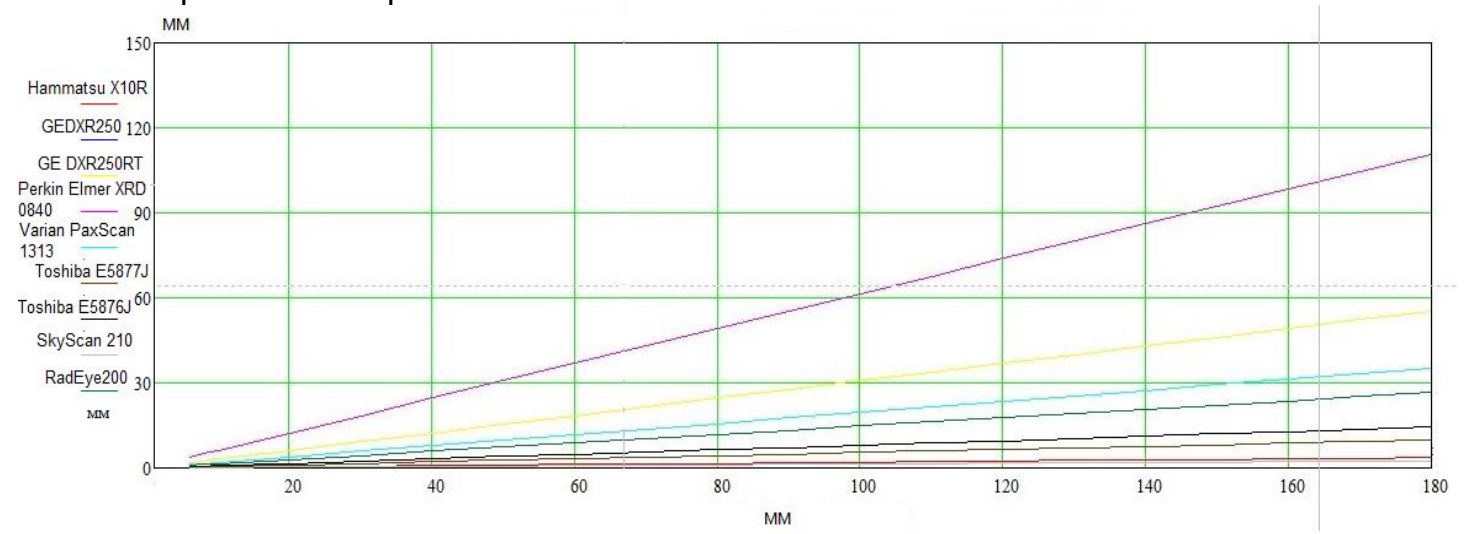

Рисунок 4 - График зависимости размера вокселя от расстояний до оси вращения

Возможность достоверной реконструкции дозового поля и, следовательно, структуры объекта диагностики существенно зависит от режима проведения томографии. Качество получаемого изображения и разрешающую способность системы зависят от правильности выбора таких параметров, как расстояние, напряжение, сила тока и др. Например, изображение, снятое при напряжении 80 кВ, более зашумленное, чем снятое при 140 кВ. Крайне важно в выборе элементов рентгенооптической системы опираться на предложенные в статье критерии.

\section{ЗАКЛЮЧЕНИЕ}

В заключении хочется отметить, что в статье получены критерии выбора элементов, их ключевые характеристики, основные зависимости параметров, показатели качества, а также рекомендации по выборе элементов рентгеновских систем. Зная поведение, полученных эмпирическим путем зависимостей параметров рентгенооптической системы, можно легко и быстро спрогнозировать величину выходной интенсивности, напряжения, силы тока на аноде, размера фокального пятна и вокселя. Ведь зная зависимость, полученную из практических данных, наиболее точно и полно описывает систему. Этот фракт очень выгодно отличает данную математическую зависимость от имеющихся теоретических средств расчета. Данная модель имеет значимость на практике, так как позволяет получать более качественные теневые изображения для построения трехмерных изображений, а также облегчает работу инженеров и конструктов по разработке новых модификаций рентгеновских систем. Предложенные критерии не только облегчат набор работ по проектированию рентгеновских систем, но станет полезным и при выборе необходимых параметров системы для конкретного рентгеновского исследования.

\section{СПИСОК ЛИТЕРАТУРЫ}

1. Landisa E., Keane D. (2010) X-ray microtomography. Materials Characterization, vol. 61, no 12, pp. 1305-1316.

2. Кокорева А.Е., И.В. Плотникова И.В., Гальцева О.В., Китаева М.В. Контроль точности результатов измерений // Ползуновский вестник / Алтайский государственный технический университет им. И. И. Ползунова (АлтГТУ). - 2016. - Т. 2, № 4. - C. 84-87

3. Гальцева О.В., Бордунов С.В., Никитина А.И. Использование электровзрывной технологии для обогащения минерального сырья // Ползуновский вестник. 2016. № 4-2. С. 181-185/

4. Чичерина Н.В. Ресурсный центр в структуре профессионального образования как основа технологической подготовки учащихся // 
Профессионально образование. Столица.- 2012. №11 - c. 44-48

5. Гаврилин А.Н., Мойзес Б.Б. Диагностика технологических систем: учебное пособие. Часть $1 /$ Томский политехнический университет. Томск: Изд-во Томского политехнического университета, 2013. - 120 с.

6. Гаврилин А.Н., Мойзес Б.Б. Диагностика технологических систем. Часть 2: учебное пособие / Томский политехнический университет. Томск: Изд-во Томского политехнического университета, 2013. - 128 с.

7. Гольдштейн А.Е., Вавилова Г.В. Технологический контроль погонной емкости электрического кабеля в условиях значительных изменений солености воды / А.Е. Гольдштейн, Г.В. Вавилова// Контроль. Диагностика. - 2013. - № 9. - с. 57-60.

8. Мазиков С.В. Метрологическое обеспечение измерителя емкости САР-10.1/ С.В. Мазиков, Г.В. Вавилова // Ползуновский вестник. -2016. - № 2. - с. 65-68.

9. Dunsmuir J., Bennett S., Fareria L., Mingino A., Sansone M. (2006) X-ray microtomographic imaging and analysis for basic research. Powder Diffraction, vol. 21, no 2, pp. 125-131

10. Baus S., Redko L., Yanushevskaya M. Xray Tomographic System Behavior Prediction Based on a Mathematical Model: IOP Conf. Ser.: Mater. Sci. Eng. 189 012016, 2017

11. Natterrer F. The Mathematics of Computerized Tomography, Münster: Classics in Applied Mathematics, Society for Industrial and Applied Mathematics, 2001
12. Li Yan. (1992) Mathematical models for radiographic exposure and their application. NDT \& $E$ International, vol. 25, no 4-5, pp. 183-189.

13. Назипов Р.А., Храмов А.С., Зарипова Л.Д. Основы радиационного неразрушающего контроля: учеб.- метод. пособие для студентов физического факультета. Казань: Изд-во КГУ. - 2008. C.6

Баус Станислав Сергеевич, аспирант, кафедра автоматики и управления (АиУ), Институт Автоматики и Электронного Приборостроения, ФГБОУ ВО «Казанский национальный исследовательский технический университет имени А. Н. Туполева-КАИ» stas.baus@yandex.ru (КНИТУ-КАИ) e-mail:

Плотникова Инна Васильевна, канд. техн. наук, доцент отделения контроля $и$ диагностики Инженерной школы неразрушающего контроля и безопасности ФГАОУ ВО «Национальный исследовательский Томский политехнический университет», e-mail: inna@tpu.ru.

Чичерина Наталия Викторовна, канд. пед. наук, доцент отделения контроля $u$ диагностики Инженерной школы неразрушающего контроля и безопасности ФГАОУ ВО «Национальный исследовательский Томский политехнический университет», e-mail: chicherina@tpu.ru 